\title{
Continued Elevation of Plasma IL-4 and IL-17 Predicts the Progression from VMC to DCM
}

\author{
Z. H. Wang $\mathbb{D}^{1}{ }^{1}$ Y. H. Liao ${ }^{1},{ }^{1}$ J. Yuan, ${ }^{1}$ X. J. Jin, ${ }^{2}$ M. Yu, ${ }^{1}$ R. Z. Chen, ${ }^{2}$ D. J. Xu, ${ }^{3}$ J. Wei, ${ }^{4}$ \\ J. Wan, ${ }^{5}$ D. C. Zhao, ${ }^{6}$ H. Y. Han, ${ }^{7}$ B. Li, ${ }^{8}$ G. Tian ${ }^{9},{ }^{9}$ G. Hu, ${ }^{10}$ and J. $\mathrm{Xu}^{11}$ \\ ${ }^{1}$ Department of Cardiology, Union Hospital, Tongji Medical College, Huazhong University of Science and Technology, Wuhan, China \\ ${ }^{2}$ Department of Cardiology, Fudan University, Zhongshan Hospital, Shanghai, China \\ ${ }^{3}$ Department of Cardiology, The First Affiliated Hospital of Nanjing Medical University, Nanjing, China \\ ${ }^{4}$ Department of Cardiology, The Second Affiliated Hospital of Xi'an Jiaotong University, Xi'an, China \\ ${ }^{5}$ Department of Cardiology, Zhongnan Hospital of Wuhan University, Wuhan, China \\ ${ }^{6}$ Department of Cardiology, The First Affiliated Hospital of Harbin Medical University, Harbin, China \\ ${ }^{7}$ Department of Cardiology, Tianyou Hospital Affiliated to Wuhan University of Science \& Technology, Wuhan, China \\ ${ }^{8}$ Department of Cardiology, The Second Hospital of Shanxi Medical University, Taiyuan 030001, China \\ ${ }^{9}$ Department of Cardiology, First Affiliated Hospital Xi'an Jiaotong University, Xi'an, Shanxi, China \\ ${ }^{10}$ Department of Cardiology, Jingzhou Central Hospital, Jingzhou, Hubei, China \\ ${ }^{11}$ Department of Cardiology, Renji Hospital, Shanghai Jiaotong University School of Medicine, Shanghai, China
}

Correspondence should be addressed to Y. H. Liao; liaoyh27@163.com

Received 10 June 2019; Accepted 5 November 2019; Published 11 January 2020

Academic Editor: Donald H. Chace

Copyright (c) 2020 Z. H. Wang et al. This is an open access article distributed under the Creative Commons Attribution License, which permits unrestricted use, distribution, and reproduction in any medium, provided the original work is properly cited.

Objective. To investigate plasma cytokines (interferon gamma, interleukin-4, and interleukin-17) in patients with viral myocarditis (VMC) and evaluate their predictive value in the progression from VMC to dilated cardiomyopathy (DCM). Methods. A prospective, multicenter, observational study included 536 patients with newly diagnosed VMC admitted in cardiology departments of 24 tertiary super specialised university-affiliated hospitals in the China registry from January 2012 to June 2016. Demographics and clinical characteristics at baseline and after three months were collected, including laboratory blood tests, ECG, echocardiography, and drug treatment in each participating site. The plasma anti-viral antibodies (Abs), anti-heart autoimmune Abs, and cytokines were detected by ELISA. Results. Of the 536 patients, 534 were included for analysis after two patients died in less than a month. The plasma levels of IFN- $\gamma$, IL-4, and IL-17 were continually higher in patients with incident DCM than in those without incident DCM at baseline, from the 1st month and the 3rd month; all had a $P$ value of $<0.0001$. There was a positive correlation between IL- 4 and LVEDd $(r=0.30, P<0.0001)$ and between IL-17 and LVEDd $(r=0.11, P=$ 0.02). When all these covariates have entered the model simultaneously, elevated IL- 4 and IL-17 were still significantly associated with DCM incidence. The RR (95\% CI) of DCM incidence were 1.04 (1.02-1.06) for IL-4 and 5.24 (2.81-9.79) for IL17. Conclusion. The continued elevation of plasma IL-4 and IL-17 in VMC patients were associated with a high incidence of DCM at three months, and these two cytokines were independent predictors for the progression from VMC to DCM.

\section{Introduction}

Viral myocarditis (VMC) is a common illness worldwide that can lead to severe complications or death in infants and young adults $[1,2]$, which is responsible for sudden death cases in young adults $(8.6 \%-12 \%)$ and $9 \%$ to $16 \%$ of newly onset dilated cardiomyopathy (DCM) $[3,4]$. The incidence of myocarditis is approximately 1.5 million cases worldwide per year [5]. Acute VMC, a precursor of DCM leading to heart failure, is a triphasic disease involving an initial viral infection, followed by autoimmune response, and finally remodelling of cardiac structure and function [6].

The pathogenesis of DCM secondary to VMC is closely associated with dysfunction of the autoimmune system. 
CD4 ${ }^{+}$Th cell subsets (Th1, Th17, and Th2) are involved in the mechanisms for the onset of VMC and DCM and the progressing from VMC to DCM [6-8]. The acute viral infection will activate the Th0 cells, and in so doing, this will initiate a cascade of events as follows: Th0 will differentiate to Th1 cells resulting in the production of IFN- $\gamma$, thus fueling myocardial cell injury. In the acute stage, Th0 will also differentiate to Th17 cells resulting in the production of IL-17. IL-17, on the one hand, works on cytotoxic T lymphocytes (CTLs) to lead to myocardial cell injury, and on the other hand, it also works on B lymphocytes to produce several anti-heart autoantibodies (AHAs). In the chronic stage, Th0 differentiates to Th2 cells causing the production of IL-4, which promotes B cells to produce AHAs. AHAs can mediate $\mathrm{Ca}^{2+}$ influx into the myocardial cells and damage them [6-12]. The objective of our study was to observe the transforming rate progressing from VMC to DCM in China and to decide if cytokines (IL-17, IL-4, and IFN- $\gamma$ ) could predict this progression.

\section{Methods}

2.1. Study Population. A total of 536 patients with newly diagnosed clinical VMC admitted in 24 cardiology departments of tertiary super specialised hospitals in China were enrolled in the registry in each participating center from January 2012 to June 2016. There were no specific exclusion criteria. A signed informed consent was obtained.

2.2. Study Design. This is a prospective, multicenter, observational study. Demographics and clinical characteristics at baseline and after three months were collected from all patients, including laboratory blood tests, ECG, echocardiography, and drug treatment at each participating site. The plasma anti-viral antibodies (Abs), anti-heart autoimmune Abs, and cytokines were detected by ELISA. VMC clinical types enrolled for the study were divided by four categories: arrhythmia type, heart failure type, acute severe type, and subclinical type according to the 1999 Chinese viral myocarditis diagnostic standard and the 2013 ESCs $[4,13]$. The following VMC clinical types were enrolled for the study:

(a) Arrhythmia type: 1 to 3 weeks after virus infection, mild precordial discomfort, palpitations, ECG reveals premature beats or tachycardia, atrioventricular block, and ST-T changes. There was increased TnI, no clinical manifestations of heart enlargement and heart failure, and gradual recovery after 1 to 2 months of treatment

(b) Heart failure type: 1-3 weeks after the virus infection, fatigue, palpitation, dyspnea and other symptoms, increased TnI, cardiac enlargement and heart failure can be associated with arrhythmia, and some patients evolved to dilated cardiomyopathy

(c) Acute severe type: 1 to 2 weeks after the virus infection, chest pain, palpitation, difficulty in breathing, occurrence of ventricular tachycardia, ventricular gallop, heart failure, cardiac enlargement and other clinical manifestations or even cardiogenic shock, with TnI significantly increased. This type of illness is dangerous, and some patients showed eruption of myocarditis and could have died within a few days or weeks due to pump failure or severe arrhythmia

(d) Subclinical type: no symptoms after virus infection and ECG detected ST-T changes which may disappear early in the morning or a few weeks later

The definition for VMC progressing to DCM is the appearance or persistence of LVEDd $\geq 5.5 \mathrm{~cm}$ and LVEF $<45 \%$ in patients given optimal medical treatment and followed up for three months [13].

2.3. Statistical Analysis. Baseline characteristics of the viral myocarditis patient cohort were summarised according to the incidence of dilated cardiomyopathy for three months follow-up. All data are presented as mean \pm SD for continuous variables, median (interquartile range) if continuous variables were skewed, and $n$ (\%) for categorical variables. Comparisons between groups were performed by the chisquare or Fisher exact test (for categorical variables) and the Student $t$-test or Mann-Whitney $U$ test (for continuous variables). Linear mixed-effect models and logistic regression models with adjustment for gender, age, and baseline echocardiography covariables were used to identify the change of cytokine status (after natural logarithmic transformation) that pose the highest risk for incident DCM. The C statistics for each risk factor were calculated to estimate the predictive values of incident DCM. A $P$ value of $<0.05$ was considered statistically significant. All statistical analyses were performed using SAS version 9.3 (SAS Institute Inc., Cary, NC).

\section{Results}

3.1. Patient Characteristics and Incident DCM. Included in the study were 536 patients of a VMC cohort, of which two patients died in less than one month. A comparison of baseline clinical and laboratory parameters between patients with and without DCM is presented in Table 1. After three months follow-up, 127 (23.78\%) newly onset DCM were recorded among 534 patients of the VMC cohort; out of the 127 patients who reached the primary endpoint, significant types were in 46 patients (36.22\%) of the acute severe type and in 62 patients $(48.82 \%)$ of the heart failure type. In comparison with patients without DCM, those patients with DCM presented at an older age, with lower LVEF, larger LVEDD, worse NYHA class, higher NT-pro-BNP levels, and an increased virus infection rate (all with $P<0.0001$ ). Baseline antibodies (against ANT, $\beta 1 \mathrm{R}, \mathrm{MHC}$, and $\mathrm{CaC}$ ) and cytokines (IFN- $\gamma$, IL-4, and IL-17) were significantly higher in patients who developed DCM as compared to those who did not develop DCM.

3.2. Prediction of Incident DCM in Patients with VMC. Univariate analysis shows all variables concerning incident DCM after three months (Table 2). Adjusted relative risks of incident DCM after three months, multivariate analysis of age, LVEF, IL-4, and IL-17 predicted the occurrence of 
TABLE 1: Comparison of baseline clinical, morphometric, and laboratory parameters between without and with DCM according to treatment response.

\begin{tabular}{|c|c|c|c|c|}
\hline & \multirow{2}{*}{$\begin{array}{c}\text { All patients } \\
\text { With follow-up } \\
(n=534)\end{array}$} & \multicolumn{2}{|c|}{ Subgroups } & \multirow[b]{2}{*}{$P$ value } \\
\hline & & $\begin{array}{l}\text { Without DCM } \\
\quad(n=407)\end{array}$ & $\begin{array}{c}\text { With DCM } \\
(n=127)\end{array}$ & \\
\hline Age (yrs), mean $\pm S D$ & $35.81 \pm 15.19$ & $32.0 \pm 13.3$ & $48.1 \pm 14.5$ & $<0.0001$ \\
\hline Male (\%) & $296(55.43 \%)$ & $211(51.84 \%)$ & $85(66.93 \%)$ & 0.0028 \\
\hline VMC subtype & & & & $<0.0001$ \\
\hline Acute severe type & $81(15.17 \%)$ & $35(8.6 \%)$ & $46(36.22 \%)$ & \\
\hline Heart failure type & $139(26.03 \%)$ & $77(18.92 \%)$ & $62(48.82 \%)$ & \\
\hline Arrhythmia type & $215(40.26 \%)$ & $211(51.84 \%)$ & $4(3.15 \%)$ & \\
\hline Subclinical type & $99(18.54 \%)$ & $84(20.64 \%)$ & $15(11.81 \%)$ & \\
\hline NYHA III-IV & $145(27.90 \%)$ & $70(17.20 \%)$ & $79(62.2 \%)$ & $<0.0001$ \\
\hline ECG with arrhythmia & $418(78.28 \%)$ & $303(74.20 \%)$ & $116(91.3 \%)$ & $<0.0001$ \\
\hline ECG with ST-T changes & $252(47.01 \%)$ & $153(37.41 \%)$ & $99(77.95 \%)$ & $<0.0001$ \\
\hline \multicolumn{5}{|l|}{ Echocardiogram } \\
\hline Left atrium $(\mathrm{cm})$ & $3.6 \pm 0.84$ & $3.3 \pm 0.6$ & $4.6 \pm 0.8$ & $<0.0001$ \\
\hline Right atrium $(\mathrm{cm})$ & $3.65 \pm 0.88$ & $3.4 \pm 0.7$ & $4.5 \pm 1.0$ & $<0.0001$ \\
\hline Left ventricle $(\mathrm{cm})$ & $5.27 \pm 1.52$ & $4.8 \pm 0.8$ & $6.7 \pm 2.2$ & $<0.0001$ \\
\hline Right ventricle $(\mathrm{cm})$ & $2.96 \pm 0.92$ & $2.9 \pm 0.9$ & $3.1 \pm 1.1$ & 0.1946 \\
\hline LVEF (\%) & $52.61 \pm 15.14$ & $58.0 \pm 12.0$ & $34.8 \pm 9.7$ & $<0.0001$ \\
\hline$E / A<1$ & $110(30.47 \%)$ & $88(29.04 \%)$ & $22(37.93 \%)$ & 0.156 \\
\hline \multicolumn{5}{|l|}{ Laboratory } \\
\hline WBC (G/L) & $7.47 \pm 2.76$ & $7.5 \pm 2.9$ & $7.5 \pm 2.3$ & 0.9646 \\
\hline $\operatorname{ALT}(\mathrm{U} / \mathrm{L})$ & $26.5(16-48.05)$ & $26.0(15.0-43.0)$ & $32.6(19.0-68.0)$ & 0.0122 \\
\hline $\mathrm{Cr}(\mu \mathrm{mol} / \mathrm{L})$ & $71.7(57.3-86.2)$ & $70.0(56.0-82.3)$ & $80.0(65.3-99.0)$ & $<0.0001$ \\
\hline Coxsackievirus B5-IgM & $357(66.85 \%)$ & $247(60.69 \%)$ & $110(86.61 \%)$ & $<0.0001$ \\
\hline Coxsackievirus B3-IgM & $344(64.42 \%)$ & $230(56.51 \%)$ & $114(89.76 \%)$ & $<0.0001$ \\
\hline Cytomegalovirus-IgM & $269(50.37 \%)$ & $166(40.79 \%)$ & $103(81.1 \%)$ & $<0.0001$ \\
\hline Enterovirus RNA & $351(65.73 \%)$ & $238(58.48 \%)$ & $113(88.98 \%)$ & $<0.0001$ \\
\hline Anti-ANT antibody & $433(81.09 \%)$ & $311(76.41 \%)$ & $122(96.06 \%)$ & $<0.0001$ \\
\hline Anti-MHC antibody & $224(41.95 \%)$ & $135(33.17 \%)$ & $89(70.08 \%)$ & $<0.0001$ \\
\hline Anti- $\beta 1 \mathrm{R}$ antibody & $287(53.75 \%)$ & $196(48.16 \%)$ & $91(71.65 \%)$ & $<0.0001$ \\
\hline Anti-CaC antibody & $263(49.25 \%)$ & $172(42.26 \%)$ & $91(71.65 \%)$ & $<0.0001$ \\
\hline CK-MB (ng/mL) & $13.8(3.9-28)$ & $14(4-30)$ & $13(1.7-17)$ & 0.1013 \\
\hline TNI-T (ng/mL) & $0.05(0-0.9)$ & $0.05(0-1.03)$ & $0.02(0.01-0.16)$ & 0.4629 \\
\hline NT-pro-BNP (pg/mL) & $300(55.8-2186)$ & $189.9(35.0-1691.0)$ & $1462.3(229.0-3042.0)$ & $<0.0001$ \\
\hline IFN- $\gamma(\mathrm{pg} / \mathrm{mL})$ & $79.24(12.99-106.33)$ & $76.48(11.00-105.56)$ & $96.95(80.70-122.57)$ & 0.0165 \\
\hline IL-4 (pg/mL) & $45.29(28.14-64.11)$ & $38.24(20.91-55.98)$ & $84.25(59.20-103.74)$ & $<0.0001$ \\
\hline IL-17 (pg/mL) & $21.19(14.73-41.88)$ & $19.44(12.68-25.11)$ & $38.81(30.42-50.42)$ & $<0.0001$ \\
\hline \multicolumn{5}{|l|}{ Medications } \\
\hline Astragalus membranaceus & $213(39.89 \%)$ & $162(39.80 \%)$ & $51(40.16 \%)$ & 0.9433 \\
\hline CoQ10 & $296(55.43 \%)$ & $229(56.27 \%)$ & $67(52.76 \%)$ & 0.4873 \\
\hline Vitamin C & $205(38.46 \%)$ & $162(39.90 \%)$ & $43(33.86 \%)$ & 0.2218 \\
\hline ACEIs/ARBs & $114(21.35 \%)$ & $87(21.38 \%)$ & $27(21.26 \%)$ & 0.9778 \\
\hline Beta blocker & $218(40.82 \%)$ & $175(43.00 \%)$ & $43(33.86 \%)$ & 0.0673 \\
\hline Amiodarone & $28(5.24 \%)$ & $20(4.91 \%)$ & $8(6.30 \%)$ & 0.5409 \\
\hline
\end{tabular}

Means \pm standard deviations and proportions of characteristics at baseline were estimated by incident DCM status. With DCM $=$ incident of dilated cardiomyopathy, compared to without DCM. 
TABLE 2: Adjusted relative risks (95\% confidence interval) of incident DCM after three months.

\begin{tabular}{|c|c|c|}
\hline Baseline characteristics & ${\text { Univariate } \text { model }^{\ddagger}}^{\ddagger}$ & 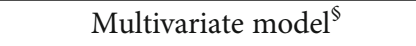 \\
\hline Age (yrs) & $1.08(1.06-1.10)^{\dagger}$ & $1.036(1.008-1.066), P=0.0002^{*}$ \\
\hline Male & $1.88(1.24-2.86)^{\dagger}$ & NS \\
\hline $\operatorname{LVEF}(\%)$ & $0.88(0.86-0.90)^{\dagger}$ & 0.895 (0.866-0.925), $P<0.0001^{\dagger}$ \\
\hline $\operatorname{Ln}(\operatorname{IFN}-\gamma)$ & $1.85(1.14-2.98)^{*}$ & NS \\
\hline $\operatorname{Ln}(\mathrm{IL}-4)$ & $1.06(1.05-1.07)^{\dagger}$ & $1.039(1.022-1.055), P<0.0001^{\dagger}$ \\
\hline $\operatorname{Ln}(\mathrm{IL}-17)$ & $3.65(2.56-5.20)^{\dagger}$ & $5.241(2.806-9.789), P<0.0001^{\dagger}$ \\
\hline Ln(NT-pro-BNP) & $1.236(1.102-1.386)^{\dagger}$ & NS \\
\hline Coxsackievirus B5-IgM (+) & $4.19(2.42-7.25)^{\dagger}$ & NS \\
\hline Coxsackievirus B3-IgM (+) & $6.75(3.68-12.37)^{\dagger}$ & NS \\
\hline Cytomegalovirus-IgM (+) & $6.23(3.83-10.13)^{\dagger}$ & NS \\
\hline Enterovirus RNA (+) & $5.73(3.18-10.33)^{\dagger}$ & NS \\
\hline Anti-ANT antibody $(+)$ & $7.53(2.99-18.96)^{\dagger}$ & NS \\
\hline Anti- $\beta 1 \mathrm{R}$ antibody $(+)$ & $4.72(3.06-7.27)^{\dagger}$ & NS \\
\hline Anti-MHC antibody $(+)$ & $2.72(1.77-4.19)^{\dagger}$ & NS \\
\hline Anti-CaC antibody $(+)$ & $3.45(2.24-5.33)^{\dagger}$ & NS \\
\hline
\end{tabular}

Ln: natural logarithmic transformation. ${ }^{*}$ Model 1: univariate model. ${ }^{\S}$ Model 2: multivariate model, all variables were entered into the model. ${ }^{\dagger} P<0.001$. ${ }^{*} P<0.05$.

TABle 3: Prediction of incident DCM in patients with VMC (RR 95\% CI).

\begin{tabular}{lc}
\hline & AUC $(95 \%$ CI $)$ \\
\hline Age (yrs) & $0.7961(0.7526-0.8395)^{\dagger}$ \\
Gender & $0.5767(0.5290-0.6244)^{*}$ \\
LVEF (\%) & $0.9092(0.8822-0.9362)^{\dagger}$ \\
UCG LV & $0.9075(0.8689-0.9461)$ \\
Ln(IL-4) & $0.8566(0.8057-0.9075)^{\dagger}$ \\
Ln(IL-17) & $0.7682(0.7223-0.8141)$ \\
Basic model (without cytokine, & $0.9155(0.8769-0.9540)$ \\
age+gender+LVEF) & $0.9344(0.8948-0.9741)^{\dagger}$ \\
Basic model+ln(IL-4)+ln(IL-17) &
\end{tabular}

DCM; the RR (95\% CI) were between 0.895 (.866-0.925) and 5.24 (2.81-9.79). Patients with elevated levels of IL-4 and IL-17 have a high risk of developing DCM in patients with VMC; on the addition of IL-4 and IL-17 to the standard model (age, sex, and LVEF), the predictive power of progression to DCM is improved. For the prediction of incident DCM in patients with VMC (Table 3 and Figure 1), IL-4 and IL-17 significantly enhanced the area under the ROC curve (AUC) in the predicted 3-month risk of DCM vs. the basic demographic model (age, sex, and LVEF). VMC patients with higher levels of IL- 4 and IL-17 have a higher risk of developing DCM. On the addition of $\ln$ (IL-4) and $\ln$ (IL-17) to the standard model (age, sex, and LVEF), the corrected area under the curve increased from 0.9155 (95\% CI, $0.8769-0.9540)$ to 0.9344 (95\% CI, 0.8948-0.9741) for predicting the incidence of DCM events.

3.3. Correlation between Dynamic Changes of Cytokines and Occurrence of DCM. The dynamic changes of cytokines and the appearance of DCM, in which patients with

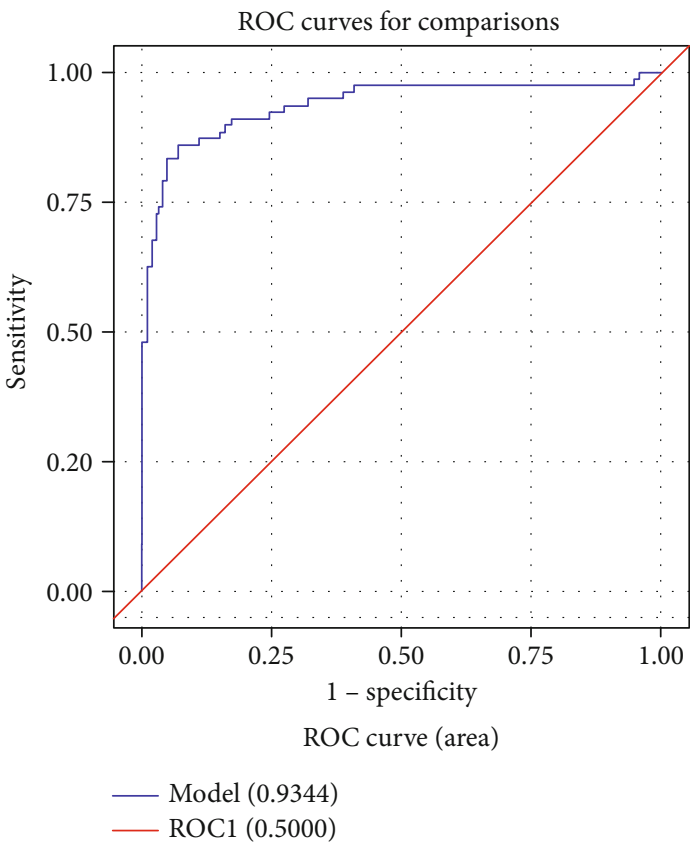

Figure 1: Predicted 3-month risk of incident DCM, age, gender, and LVEF+ln(IL-4) $+\ln ($ IL-17).

VMC who presented with high levels of IFN- $\gamma$, IL-4, and IL-17 had a higher risk of developing DCM, are summarised in Table 4. Dynamic observation showed that the levels of plasma IFN- $\gamma$, IL-4, and IL-17 were continually higher in patients with incident DCM than in those without DCM at baseline, the 1st month, and the 3rd month (all $P<0.0001$ ) as shown in the fit plot in Figure 2. For the relationship between cytokine (IFN- $\gamma$, IL-4, and IL-17) levels and echocardiographic parameters (LV), after adjusting for age, gender, 
TABLE 4: Longitudinal change of IFN- $\gamma$, IL-4, and IL-17 (pg/ml) levels in VMC patients with the incident of DCM.

\begin{tabular}{|c|c|c|c|c|c|c|c|}
\hline & & hout DCM ( & & & ith DCM $(n$ & & \\
\hline & Median & 1st quartile & 3rd quartile & Median & 1st quartile & 3rd quartile & $P$ value \\
\hline IFN- $\gamma$ & & & & & & & \\
\hline Baseline & 76.5 & 11.0 & 105.6 & 97.0 & 80.7 & 122.6 & 0.0165 \\
\hline 1st month & 79.4 & 18.8 & 116.8 & 137.4 & 109.7 & 161.8 & $<.0001$ \\
\hline 3rd month & 77.6 & 12.3 & 115.0 & 173.9 & 126.5 & 204.7 & $<.0001$ \\
\hline$P$ for trend & & 0.1334 & & & $<.0001$ & & \\
\hline IL-4 & & & & & & & \\
\hline Baseline & 38.35 & 21.05 & 56.57 & 84.25 & 59.2 & 103.74 & $<0.0001$ \\
\hline Visit 1 (1st month) & 34.04 & 19.1 & 55.35 & 92.3 & 64.85 & 113.65 & $<0.0001$ \\
\hline Visit 2 (3rd month) & 27.3 & 17.15 & 53.58 & 113.04 & 79.43 & 139.19 & $<0.0001$ \\
\hline$P$ for trend & & 0.5157 & & & $<0.0001$ & & \\
\hline IL-17 & & & & & & & \\
\hline Baseline & 19.32 & 12.63 & 24.72 & 38.81 & 30.42 & 50.42 & $<0.0001$ \\
\hline Visit 1 (1st month) & 18.06 & 12.55 & 20.64 & 43.67 & 37.25 & 50.1 & $<0.0001$ \\
\hline Visit 2 (3rd month) & 16.9 & 12.67 & 19.16 & 51.68 & 44.08 & 59.27 & $<0.0001$ \\
\hline$P$ for trend & & 0.1348 & & & 0.0235 & & \\
\hline
\end{tabular}

Data are expressed as median (1st Quartile-3rd Quartile). $P$ for trend means the mixed-effect model. Detecting three times for the sample deciding if there is longitudinal change or not.

and LVEF as well as NT-pro-BNP, IL-4 and IL-17 showed similar significant positive correlations with UCG LV, and the highest Pearson correlation was in IL-4 (correlation coefficient $r=0.30177, P=<.0001$ ) followed by IL-17 (correlation coefficient $r=0.11218, P=0.0167$ ). The mean LV size for patients with DCM was $6.7 \pm 2.2 \mathrm{~cm}$, and the mean LVEF was $34.8 \pm 9.7 \%$, which were all statistically significant with the $P$ value of $<0.0001$. There was no relationship between baseline LV and $\ln (\mathrm{IFN}-\gamma), r=0.09380, P=0.1449$. Therefore, the continued elevation of IL-4 and IL-17 can predict the incidence of DCM.

\section{Discussion}

The present study portrays the incidence of dilated cardiomyopathy from 534 patients who were diagnosed with having clinical viral myocarditis during a mean follow-up of three months, during which 127 (23.78\%) patients reached the primary endpoint (incident DCM); several studies revealed different percentages implicating myocarditis of newly onset dilated cardiomyopathy cases $[3,4,14]$. Here, we first achieved the acquisition of Chinese data about the transforming rate, which gave us a clear picture from the Chinese registry perspective.

Moreover, we further explored the role of cytokines in the progression of myocarditis to develop dilated cardiomyopathy. Although it was found that Th cells and related cytokines exert an important function in the progress of VMC to DCM, the cytokines' clinical significance as predictors of incident DCM was scantly investigated. Through a multivariate model, four variables were found to be associated with the progression to DCM, and these were age, LVEF, IL-4, and IL-17. On further analysis, IL-4 and IL-17 significantly improved the area under the ROC curve (AUC) in predicting the 3-month risk of DCM vs. the basic demographic model (age, sex, and LVEF). Increased levels of IFN- $\gamma$ in the current study did not present any significant function in predicting progression from VMC to DCM. The dual role of IFN- $\gamma$ can be in VMC pathogenesis, wherein in one hand, when increased it mediates myocardial cell injury, and on the other hand, it inhibits viral replication.

Furthermore, 15 patients out of 99 with subclinical types of VMC were observed progressing to DCM, which was expected in the advanced stages of the disease. A key point to be emphasised is that in developing DCM, the autoimmune mechanism plays a substantial role and not the severity of the patient's baseline condition. So, patients of this type require attention if IL- 4 and IL-17 levels are high even when their symptoms are mild.

The baseline UCG left ventricular dimension could not predict the incident DCM. Then, we assessed the correlation between LV dimension and cytokines, where we found positive associations between IL- 4 or IL-17 and UCG LV dimension. Despite the above fact, there was no correlation between IFN- $\gamma$ and UCG LV dimension. Both dynamic changes of IL-4, IL-17, and UCG LV dimension correlations indicated that continued elevation of plasma IL-4 and IL-17 could predict the progression from VMC to DCM.

\section{Limitations}

The present study is based on a multicenter observational registry of VMC patients. The critical limitation of this registry is that it lacks endomyocardial biopsy because of the nature of the test as an invasive clinical tool, and it is challenging for patients to accept in China. 


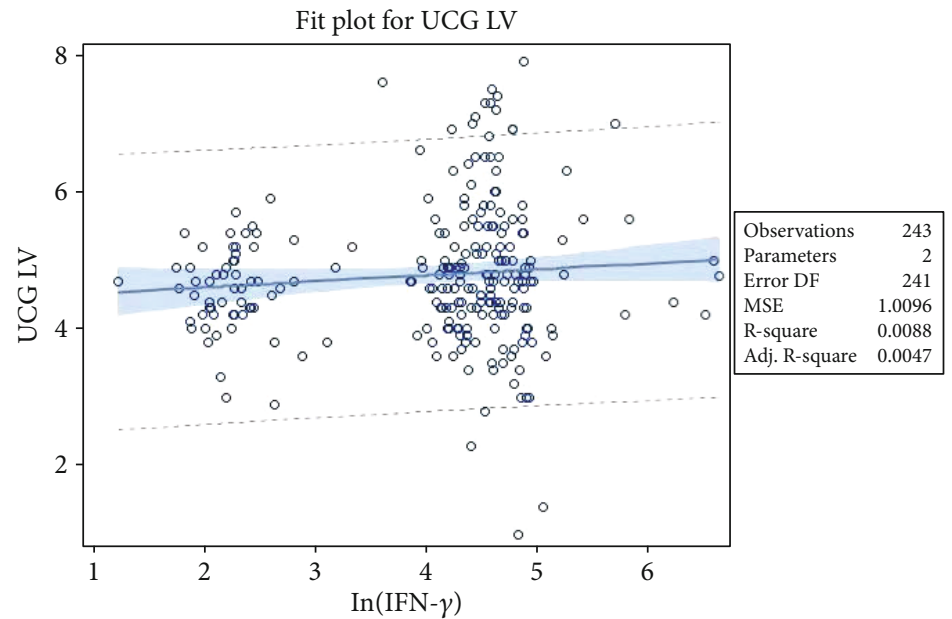

Fit $\quad \square 95 \%$ confidence limits

- - - 95\% prediction limits

(a)

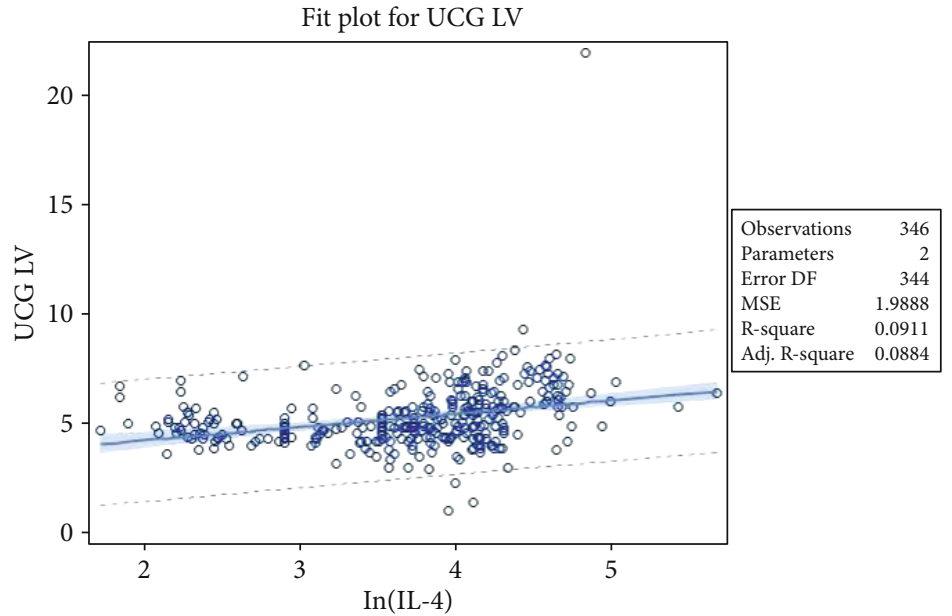

- Fit $\square 95 \%$ confidence limits

- - - 95\% prediction limits

(b)

Figure 2: Continued. 


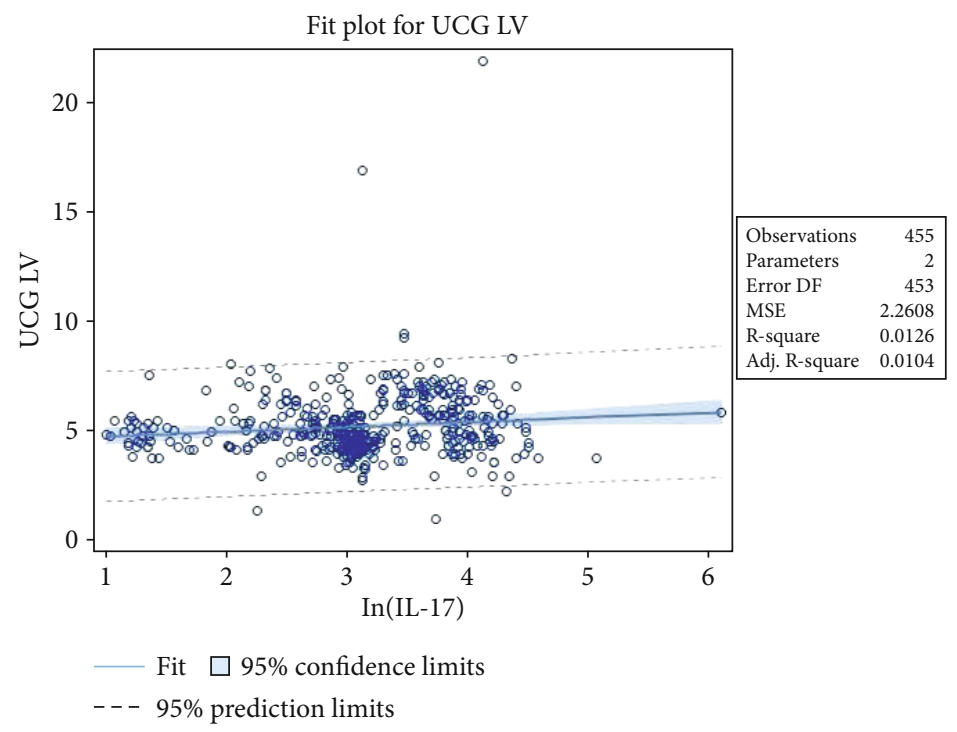

(c)

FIGURE 2: Correlation between baseline echocardiographic parameters (LV) and IFN- $\gamma$, IL-4, and IL-17. (a) No relationship was seen between baseline LV and $\ln ($ IFN- $\gamma$ ), $r=0.09380, P=0.1449$. (b and c) IL-4 and IL-17 showed similar significant positive correlations with UCG LV, with the highest for IL-4 (correlation coefficient $r=0.30177, P \leq .0001$ ) and IL-17 (correlation coefficient $r=0.11218, P=0.0167$ ).

\section{Conclusion}

The rate of transformation from VMC to DCM was about $23.78 \%$ in China, which was in concordance with other observational studies from other countries. The continued elevation of IL-4 and IL-17 in VMC patients was associated with a high incidence of DCM. Since detection for plasma IL-4 and IL-17 is not that complicated, they may serve as significant predictors for VMC evolving to DCM.

\section{Key Points}

Question. What is the role of cytokines (IL-4, IL-17, and IFN- $\gamma$ ) in predicting progression from VMC to DCM?

Findings. In this prospective multicenter observational study of 536 patients hospitalized with clinical VMC, elevated levels of plasma IL-4 and IL-17 were associated with a high incidence of DCM at 3 months, and these two cytokines were the independent predictors for the progression from VMC to DCM.

Meaning. Inflammation through inflammatory cytokines is essential for the progression of VMC to DCM. IL-4 and IL17 are significant predictors of incident DCM.

\section{Data Availability}

The deidentified participant data are accessible by contacting the corresponding author Yuhua Liao via liaoyh27@163.com.

\section{Disclosure}

An earlier version of this article prepared by J. Yuan was presented as an abstract presentation in the European Heart Journal, 2017.

\section{Conflicts of Interest}

All authors declared that there is no conflict of interest.

\section{Authors' Contributions}

Professor Liao Yuhua had full access to all the data in the study and took full responsibility for the integrity of the data and the accuracy of the data analysis. Professor Liao Yuhua also contributed in (i) study concept and design; (ii) acquisition, analysis, or interpretation of data; and (iii) critical revision of the manuscript for important intellectual content.

\section{Acknowledgments}

This work was supported by the National Science and Technology Support Program (2011BAI11B00 and 2011BAI11B23) and the National Natural Science Foundation of China (81470502 and 81400283). Study hospitals of the Myocarditis \& Cardiomyopathy Collaborative Group in China are listed as follows: Union Hospital, Huazhong University of Science and Technology, Wuhan; Zhongshan Hospital, Fudan University, Shanghai; The First Affiliated Hospital of Nanjing Medical University, Nanjing; Zhongnan Hospital, Wuhan University, Wuhan; The Second Affiliated Hospital of Xi'an Jiaotong University, Xi'an; Cardiovascular Hospital of Shanxi Province, Taiyuan; The First Hospital of Wuhan, Wuhan; Xiangyang Center Hospital, Xiangyang; Jingzhou Center Hospital, Jingzhou; The Affiliated Hospital of Harbin Medical University, Harbin; The First Affiliated Hospital of Xi'an Jiaotong University, Xi'an; The Affiliated Hospital of Jining Medical University, Jining; Shiyan People's Hospital, Shiyan; The First Affiliated Hospital of Three Gorges University, Yichang; The First Affiliated Hospital of Zhengzhou University, Zhengzhou; The Affiliated Tianyou 
Hospital of Wuhan University of Science and Technology, Wuhan; Henan Provincial People's Hospital, Zhenzhou; Renji Hospital of Shanghai Jiaotong University, Shanghai; The Second Affiliated Hospital of Harbin Medical University, Harbin; Tongji Hospital, Huazhong University of Science and Technology, Wuhan; The First Affiliated Hospital of Guanxi Medical University, Naning; and Shandong Provincial Hospital, Jinan.

\section{References}

[1] X. Zhai, B. Bai, B. Yu et al., "Coxsackievirus B3 induces autophagic response in cardiac myocytes in vivo," Biochemistry, vol. 80, no. 8, pp. 1001-1009, 2015.

[2] T. Yajima and K. U. Knowlton, "Viral myocarditis: from the perspective of the virus," Circulation, vol. 119, no. 19, pp. 2615-2624, 2009.

[3] C. Chrysohoou, C. K. Antoniou, A. Stillman, O. Lalude, T. Henry, and S. Lerakis, "Myocardial fibrosis detected with gadolinium delayed enhancement in cardiac magnetic resonance imaging and ventriculoarterial coupling alterations in patients with acute myocarditis," Hellenic Journal of Cardiology, vol. 57, no. 6, pp. 449-454, 2016.

[4] A. L. Caforio, S. Pankuweit, E. Arbustini et al., "Current state of knowledge on aetiology, diagnosis, management, and therapy of myocarditis: a position statement of the European Society of Cardiology Working Group on Myocardial and Pericardial Diseases," European Heart Journal, vol. 34, no. 33, pp. 2636-2648, 2013.

[5] S. Heymans, U. Eriksson, J. Lehtonen, and L. T. Cooper Jr., "The quest for new approaches in myocarditis and inflammatory cardiomyopathy," Journal of the American College of Cardiology, vol. 68, no. 21, pp. 2348-2364, 2016.

[6] J. Yuan, M. Yu, Q. W. Lin et al., "Th17 cells contribute to viral replication in coxsackievirus B3-induced acute viral myocarditis," Journal of Immunology, vol. 185, no. 7, pp. 4004-4010, 2010.

[7] H. Tang, Y. Zhong, Y. Zhu, F. Zhao, X. Cui, and Z. Wang, "Low responder $\mathrm{T}$ cell susceptibility to the suppressive function of regulatory $\mathrm{T}$ cells in patients with dilated cardiomyopathy," Heart, vol. 96, no. 10, pp. 765-771, 2010.

[8] J. Yuan, A. L. Cao, M. Yu et al., "Th17 cells facilitate the humoral immune response in patients with acute viral myocarditis," Journal of Clinical Immunology, vol. 30, no. 2, pp. 226-234, 2010.

[9] N. R. Rose, "Viral myocarditis," Current Opinion in Rheumatology, vol. 28, no. 4, pp. 383-389, 2016.

[10] G. C. Baldeviano, J. G. Barin, M. V. Talor et al., "Interleukin$17 \mathrm{~A}$ is dispensable for myocarditis but essential for the progression to dilated cardiomyopathy," Circulation Research, vol. 106, no. 10, pp. 1646-1655, 2010.

[11] T. Yamashita, T. Iwakura, K. Matsui et al., "IL-6-mediated Th17 differentiation through RORgammat is essential for the initiation of experimental autoimmune myocarditis," Cardiovascular Research, vol. 91, no. 4, pp. 640-648, 2011.

[12] D. Fairweather and N. R. Rose, "Inflammatory heart disease: a role for cytokines," Lupus, vol. 14, no. 9, pp. 646-651, 2005.
[13] X.-p. Zhang, S.-c. Zhang, and C.-1. Huang, "Effects of soil fauna on litter decomposition," Chinese Geographical Science, vol. 11, no. 3, article 27, pp. 283-288, 2001.

[14] R. Dennert, H. J. Crijns, and S. Heymans, "Acute viral myocarditis,” European Heart Journal, vol. 29, no. 17, pp. 2073-2082, 2008. 


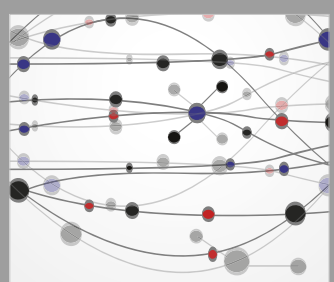

The Scientific World Journal
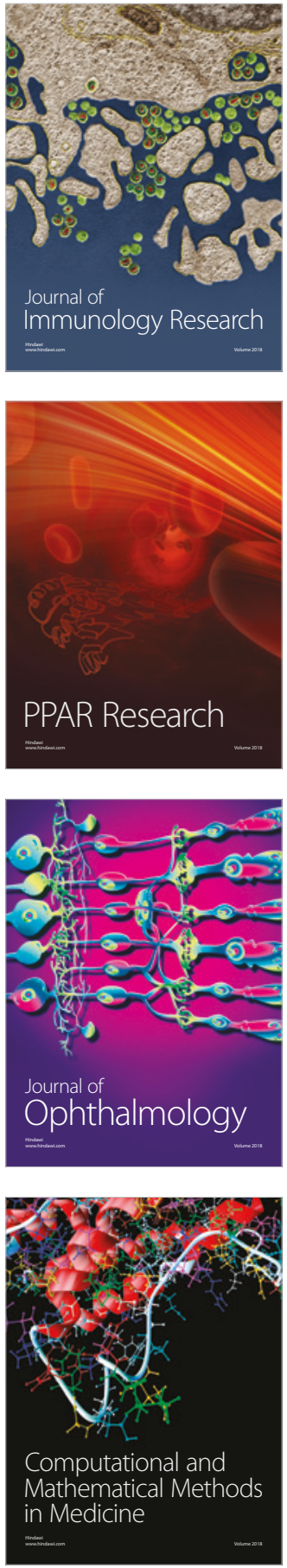

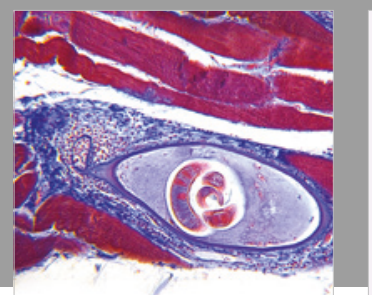

Gastroenterology Research and Practice

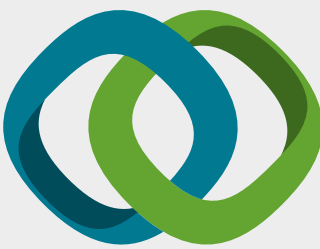

\section{Hindawi}

Submit your manuscripts at

www.hindawi.com
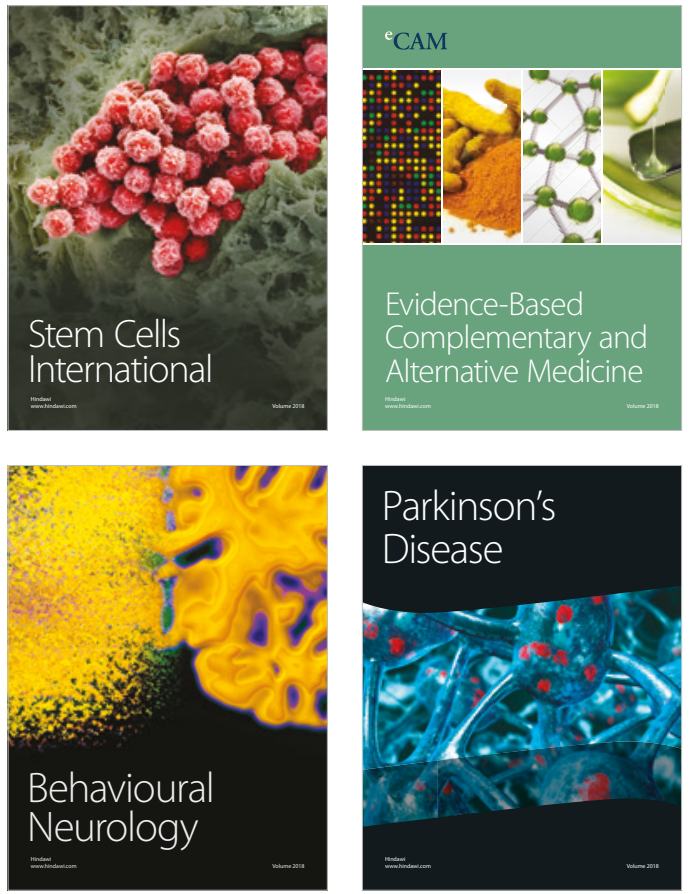

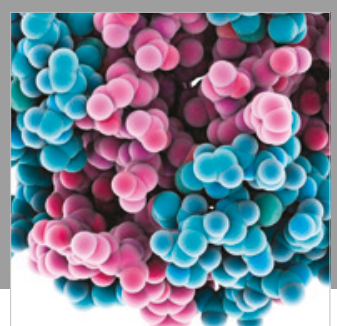

ournal of

Diabetes Research

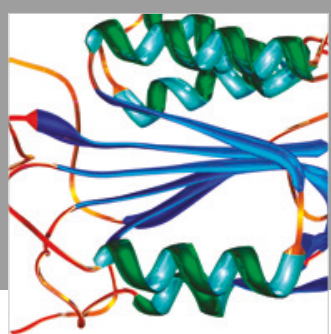

Disease Markers
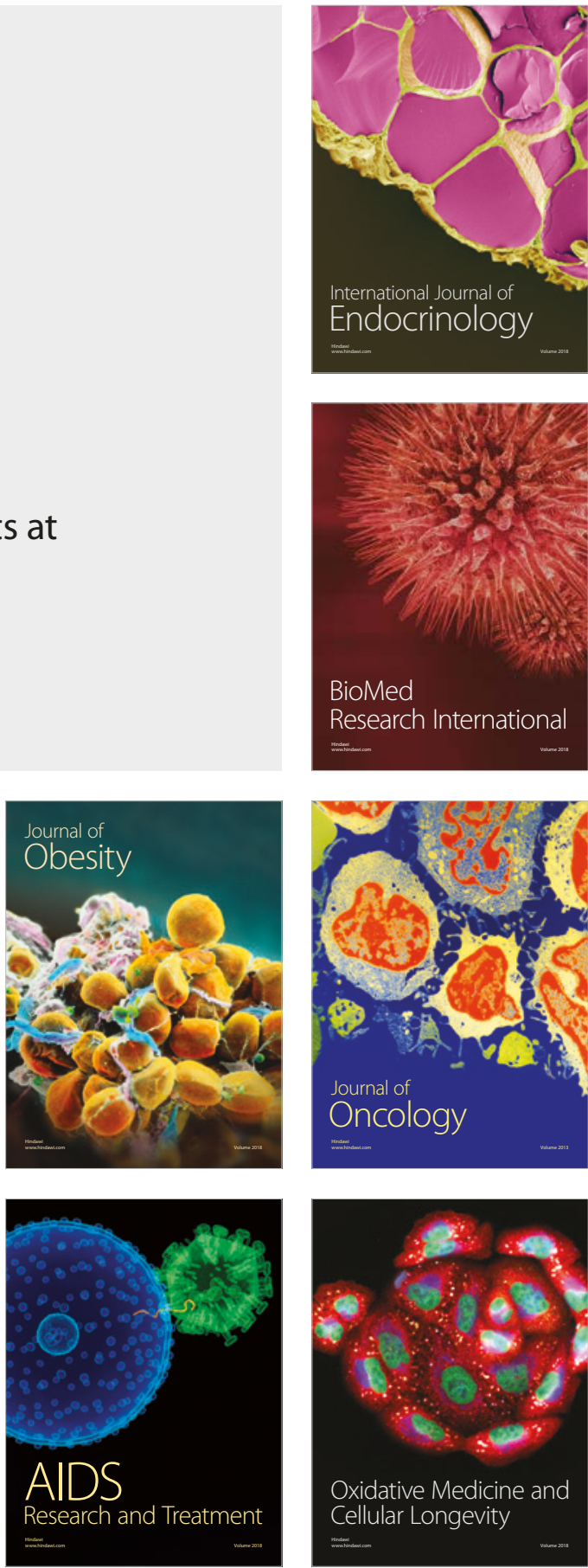